\title{
Classification of Centrilobular Emphysema Based on CT-Pathologic Correlations
}

\author{
Mamoru Takahashi ${ }^{1,2}$, Gen Yamada ${ }^{*}, 1$, Hiroyuki Koba $^{3}$ and Hiroki Takahashi ${ }^{1}$ \\ Journal \\ ${ }^{1}$ Third Department of Internal Medicine, Sapporo Medical University, School of Medicine, South-1 West-16, Chuo-ku, \\ Sapporo 060-8543, Japan \\ ${ }^{2}$ Department of Respirology, NTT East Corporation Sapporo Hospital, South-1 West-15, Chuo-ku, Sapporo 060-0061, \\ Japan \\ ${ }^{3}$ Department of Respiratory Medicine, Teine Keijin-kai Hospital, Teine-ku, Maeda 1-12, Sapporo 006-0811, Japan
}

Respiratory Medicine

\begin{abstract}
Introduction: Centrilobular emphysema (CLE) is recognized as low attenuation areas (LAA) with centrilobular distribution on high-resolution computed tomography. The LAA often exhibit a variety of shape or sharpness of border. This study was performed to elucidate the relationship between morphological features of LAA and pathological findings in CLE.

Materials and Methods: The inflated-fixed lungs from 50 patients with CLE (42 males, 8 females; 14 operated, 36 autopsied) were examined by a method of CT-pathologic correlations that consisted of three steps. The first, CT images of the sliced lungs of the inflated-fixed lung specimens were examined on the shape and the peripheral border of each LAA. The second, the sliced lungs were radiographed in contact with high magnification. The third, the surface of the sliced lungs was observed by using stereomicroscopy. The views at low magnification of stereomicroscope were compared with the radiographs and the $\mathrm{CT}$ images of the same sample.

Results: Using CT-pathologic correlations, LAAs of CLE were classified into three types as follows; round or oval shape with well-defined border (Type A), polygonal or irregular shape with ill-defined border and less than $5 \mathrm{~mm}$ in diameter (Type B), and irregular shape with ill-defined border and $5 \mathrm{~mm}$ or over in diameter (Type C). Type A, Type B and Type C LAA were mainly related to dilatation of bronchioles, destruction of proximal part of alveolar ducts, and destruction of distal part of alveolar ducts, respectively. Type A, Type B and Type C were dominant LAA in 5 (10\%), 29 (58\%) and 12 (24\%) patients, respectively. However, remained 4 patients $(8 \%)$ did not show dominant LAA type.
\end{abstract}

Conclusion: Morphological features of LAA in CLE may depend on dilatation or destruction of certain parts of the secondary lobule. Type B LAA was the commonest type in CLE.

Keywords: Low attenuation area, computed tomography, radiograph, stereomicroscopy.

\section{INTRODUCTION}

Pulmonary emphysema is a major part of COPD characterized by airflow limitation that is not fully reversible [1]. It is associated with an abnormal inflammatory response of the lung to noxious particles or gases. Using highresolution computed tomography (HRCT), pulmonary emphysema is generally classified into three types as follows; centrilobular, panlobular and paraseptal emphysema $[2,3]$.

Centrilobular emphysema (CLE) is the commonest type of pulmonary emphysema and is closely related with cigarette smoking. Low attenuation area (LAA) located in centrilobular area is detected with sensitivity, specificity and accuracy of $88 \%, 90 \%$, and $89 \%$ in HRCT, respectively [4].

*Address correspondence to this author at the Third Department of Internal Medicine, Sapporo Medical University, School of Medicine, Chuo-ku South 1 West 16, Sapporo, 060-8543, Japan; Tel: 81-11-611-2111; Fax: 81-11613-1543; E-mail: gyamada@sapmed.ac.jp
In chest HRCT, LAA often exhibit a variety of shape or sharpness of border in patients with CLE. Some patients with CLE show LAA with well-defined border, while others do not. In addition, some patients with CLE show LAA with coalescence, whereas others do individually. Therefore, CLE is not a single morphological feature defined only as the enlargement or destructions of alveolar structures. Such morphological heterogeneity on CT image is thought to be based on underlying pathological changes. However, morphological features of LAA have not been fully investigated.

To address this problem, we performed CT-pathologic correlations using inflated-fixed lung having CLE lesions. This method enables precise stereomicroscopic observations for CLE lesions on sliced lung specimens, because lung specimens are fixed in a fully inflated condition. Magnification radiography of inflated-fixed lung specimen provide ideal radiographic images for correlation with the pathologic features displayed by conventional microscopic correlation. By using this method, several pulmonary 
diseases have been examined in relation to anatomy of the secondary pulmonary lobule [5-10]. CT-pathologic correlations explain the difference of the lesions in their relation to the airway structure and are useful to understand not only small lung nodules but also emphysematous lesions $[7,8]$. This study was performed to elucidate the relationship between morphological features of LAA and pathological findings in CLE based on CT-pathologic correlations.

\section{MATERIALS AND METHODS}

\section{Materials}

This study was conducted October 2002 through March 2007. We examined inflated-fixed lungs that we prepared between April 1986 and March 1990. The lung materials were obtained from 50 patients (42 males, 8 females; 14 operated, 36 autopsied). All patients had smoking history. CLE was diagnosed by using CT images and confirmed by pathological examination. Informed consent for the CTpathologic correlations was obtained from the patients who underwent operation and the families of the patients who underwent autopsy.

The lung materials available for analysis were as follows; entire lungs from 3 patients, right lungs from 9 patients, left lungs from 20 patients, right upper lobes from 5 patients, right middle lobes from 3 patients, right lower lobes from 6 patients and left upper lobes from 4 patients.

The materials that complicated with other pulmonary diseases including infectious diseases, interstitial pneumonia, collagen diseases, cystic diseases or neoplastic diseases were excluded from this study.

This study was approved by the Institutional Review Board of Sapporo Medical University.

\section{Preparation of Inflated-Fixed Lung}

Inflated-fixed lung specimens were prepared by the method described by Markarian and Dailey [11] as follows. The lungs or lobes were fixed in distention for 2 days in a fixative fluid containing polyethylene glycol $400,95 \%$ ethyl alcohol, $37 \%$ formaldehyde, and water in proportions of $10: 5: 2: 3$

Next, the lungs were dried by forcing air through the main stem bronchus at a pressure of $10 \mathrm{cmH}_{2} \mathrm{O}$ and then cut into axial or frontal slices with $10 \mathrm{~mm}$ thickness. Number of the sliced lungs was 15 to 20 slices per each lobe.

\section{CT-Pathologic Correlations of Inflated-Fixed Lung}

CT-pathologic correlations consisted of 3 steps as follows. The first, CT images of the sliced lungs of the inflated-fixed lungs were obtained on a High Speed Advantage scanner (GE, USA) using 1-mm collimation with bone algorithm at $120 \mathrm{kVp}, 80 \mathrm{~mA}$. The sliced lungs were imaged at the window width of $1000 \mathrm{HU}$ and the window level of $-700 \mathrm{HU}$. CT images of the sliced lungs were examined on the shape and the peripheral border of each LAA.

The second, the sliced lungs were radiographed in contact with a fine resolution and high contrast film with high magnification (Fuji FR for soft $\mathrm{X}$ ray, Fuji photo film, Tokyo) at $14 \mathrm{kVp}, 450 \mathrm{~mA}$ and $60 \mathrm{~cm}$ focus-film distance with soft $X$ ray apparatus (CSM-2, Softex, Tokyo). Number of the radiographs was 3 to 5 per each sliced lung. LAA detected in the CT image of the sliced lung was compared with the radiograph image.

The third, the surface of the sliced lung was observed by using stereomicroscopy. The views at low magnification of stereomicroscope were compared with the radiograph and the CT images of the same sample. The stereomicroscopic images were used for confirmation of relationship between CLE lesions and peripheral lung structures including terminal and respiratory bronchiole, alveolar duct and sac. The location of CLE lesions in the sliced lung was examined about the distribution in a secondary lobule.

As the size of secondary lobule defined by Reid [12] is about $10 \mathrm{~mm}$ in diameter, an early CLE lesion is located in the secondary lobule. Therefore, we examined LAA within $10 \mathrm{~mm}$ in diameter.

\section{Visual Assessment}

Correlations of CT findings with radiographic and stereomicroscopic findings in the sliced lung specimens were performed independently by two chest physicians who are experts for chest image diagnosis (M.T. and G.Y.). When there was a conflict in evaluation, they discussed the problem together to reach a consensus.

\section{RESULTS}

\section{Macroscopic Feature of Cut Surface of Inflated-Fixed Lungs of CLE}

The cut surface of the inflated-fixed lungs showed emphysematous foci with patchy distribution (Fig. 1). Carbon deposits were discretely or aggregately located in bronchiolar and/or alveolar structures in the cut surface of the inflated-fixed lungs. Discrete carbon deposits were mainly distributed along bronchiolar and/or alveolar structures.

\section{Classification of LAA in Inflated-Fixed Lungs}

CT images of the sliced lung of inflated-fixed lungs showed several types of LAA with centrilobular distribution. In the consideration with the shape of LAA and its border with surrounding parenchyma, three types of LAA were recognized in CT (Fig. 2).

The first was LAA which were round or oval shape with well-defined border. This type of LAA was surrounded by normal lung density in CT images. The size of LAA was less than $5 \mathrm{~mm}$ in diameter in radiograph and stereomicroscopic images. They were pathologically related to dilatation of terminal and/or respiratory bronchiole, according to the comparison between radiographic and stereomicroscopic images. The border of LAA was formed by the membranous structures of the bronchiole. Destruction of the alveolar ducts and alveoli around the CLE lesions were relatively mild in stereomicroscopic images.

The second was LAA which were polygonal or irregular shape with ill-defined border. This type of LAA was surrounded by normal lung density in CT images. The size 

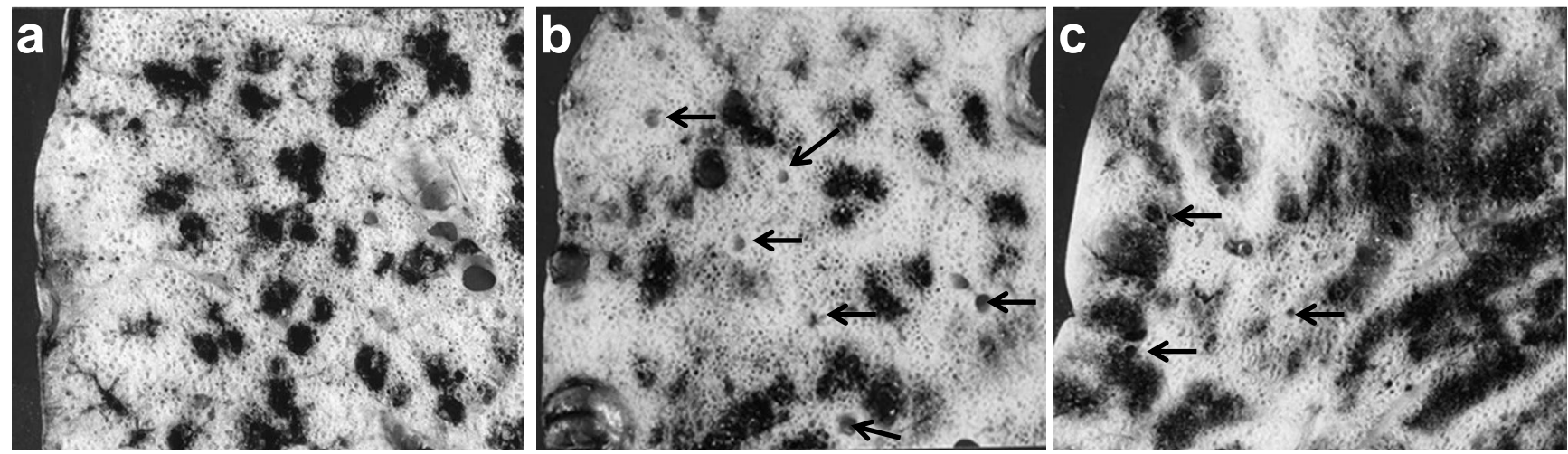

Fig. (1). Cut surfaces of Inflation-fixed lung specimens. CLE lesions with carbon deposits can be seen with patchy distribution. CLE lesions are located in independent $(\mathbf{a}, \mathbf{b})$ or coalesced feature (c). Carbon deposits were observed mainly in bronchiolar structure (a) or alveolar structure $(\mathbf{b}, \mathbf{c})$. Arrow shows bronchiolar structures without carbon deposits.
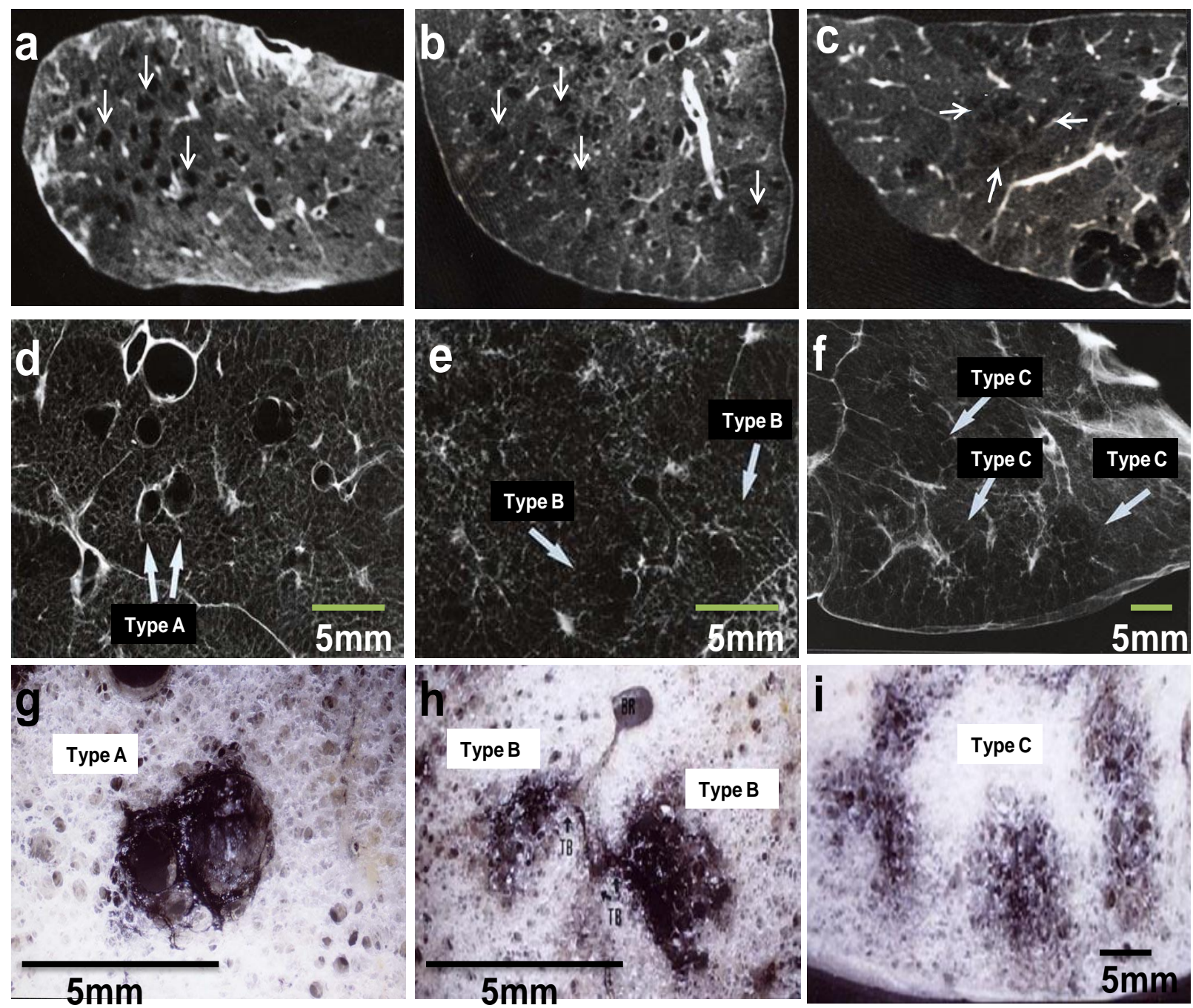

Fig. (2). High-resolution CT, radiograph and stereomicroscopic images of inflated-fixed lungs. High-resolution CT (a, b, c), radiograph (d, e, f) and stereomicroscopic images $(\mathbf{g}, \mathbf{h}, \mathbf{i})$ were obtained from specimens of $5 \mathrm{~mm}$-thick slice of inflated-fixed lungs. Type A LAA was round or oval shape with well-defined border $(\mathbf{a}, \mathbf{d})$. The size was less than $5 \mathrm{~mm}$ in diameter. Type B LAA was polygonal or irregular shape with ill-defined border $(\mathbf{b}, \mathbf{e})$. The size was less than $5 \mathrm{~mm}$ in diameter. Type C LAA was irregular shape with ill-defined border, coalescing with each other $(\mathbf{c}, \mathbf{f})$. The size was $5 \mathrm{~mm}$ or over in diameter. Emphysematous lesions are located in a centrilobular site with carbon deposits $(\mathbf{g}, \mathbf{h}, \mathbf{i})$. The series of image $\mathbf{a}-\mathbf{d}-\mathbf{g}, \mathbf{b}-\mathbf{e}-\mathbf{h}$ and $\mathbf{c - f - i}$ were taken from same specimen, respectively. In addition, $\mathbf{d}-\mathbf{g}, \mathbf{e}-\mathbf{h}$ and $\mathbf{f}-\mathbf{i}$ show one by one correlations, respectively. TB: terminal bronchiole. 
was less than $5 \mathrm{~mm}$ in diameter in radiograph and stereomicroscopic images. They were related to destruction of proximal portion of alveolar ducts, according to the comparison between radiographic and stereomicroscopic images. The destruction of alveolar ducts and/or alveoli in a secondary lobule was moderate, which was limited in the center of the secondary lobule in stereomicroscopic images. There was no bordering structure between the CLE lesion and surrounding lung parenchyma.

The third were LAA with irregular shape with ill-defined border, which were coalesced with each other and more irregular in shape than the second type. The size was more than $5 \mathrm{~mm}$ in diameter. Although this type of CLE was similar to panlobular emphysema, LAA with centrilobular distribution were partly recognized in each sliced lung. They were related to destruction of distal alveolar ducts and/or alveoli, according to the comparison between radiologic and stereomicroscopic images. In addition, the difference of CT densities between the LAA and surrounding lung parenchyma was relatively small. The destruction of alveolar structures in a secondary lobule was relatively severe. There was no bordering structure between the CLE lesion and surrounding lung parenchyma.

In consequence, based on our morphological observation using CT-pathologic correlations, we classified LAA within $10 \mathrm{~mm}$ in diameter into three types as follows.

Type A LAA; round or oval-shaped LAA with welldefined border

Type B LAA; polygonal or irregular-shaped LAA with ill-defined border, less than $5 \mathrm{~mm}$ in diameter

Type C LAA; irregular-shaped LAA with ill-defined border, $5 \mathrm{~mm}$ or over in diameter

\section{Frequency of Type A, Type B and Type C LAA}

In 50 patients, we examined LAA of each patient and determined dominant LAA type in the sliced lungs. A dominant LAA type was defined as a type occupied more than $50 \%$ in total number of LAA in each slice of the inflated-fixed lungs.

In most cases, one type of LAA was observed. As a result, $5(10 \%), 29(58 \%)$ and $12(24 \%)$ patients showed Type A, Type B and Type C LAA, respectively. Remained 4 patients $(8 \%)$ did not meet our definition of dominant LAA type, those contained three LAA types. In all lobes, Type B LAA was the commonest type in CLE (Table 1).

Table 1. Number of lobes with Type A, Type B and Type C LAA

\begin{tabular}{|c|c|c|c|c|c|}
\hline & RU & $\mathbf{R M}$ & $\mathbf{R L}$ & $\mathbf{L U}$ & $\mathbf{L L}$ \\
\hline \hline Type A & 2 & 3 & 4 & 6 & 5 \\
\hline Type B & 12 & 10 & 11 & 14 & 12 \\
\hline Type C & 3 & 2 & 3 & 7 & 6 \\
\hline
\end{tabular}

There was no significant difference in lobar distribution of Type A, Type B and Type C LAA. RU: right upper lobe, RM: right middle lobe, RL: right lower lobe, LU: left upper lobe, LL: left lower lobe.

\section{DISCUSSION}

Pulmonary emphysema involves predominantly the alveoli, however a significant amount of damage of small airways is also present [13]. In CLE, pathological changes are composed of preferential loss of alveolar septa and respiratory and terminal bronchioles at the center of secondary lobules. The clinical diagnosis of CLE is usually based on CT findings, reflecting pathologic evidences. CTpathologic correlations fill a gap between $\mathrm{CT}$ imaging and pathological evidences.

In this study, we examined morphological features of LAA by a method of CT-pathologic correlation using inflated-fixed lung specimens obtained from operated or autopsied patients those involved with CLE. Morphological features of LAA, reflected by CLE lesions, may be a clue for pathogenesis of CLE.

We were able to identify three types of LAA, owing to the predominant site of CLE lesions in the secondary lobule. Type A LAA was mainly composed of an extension of the terminal and/or respiratory bronchioles, forming a welldefined border. In patients with Type A LAA, pulmonary function tests including diffusion capacity may be mild, because alveoli and/or alveolar ducts were not so much destructed in our observations.

Type B and Type C LAA had ill-defined border, which was formed by destruction of alveolar structures. Type B LAA was mainly localized individually in the center of the secondary lobule, whereas Type C LAA was often extended to the entire secondary lobule. Moreover, Type C LAA coalesced each other and formed larger one than Type B LAA. This explains that Type C LAA formed irregular shape with ill-defined boarder. However, it remains unknown whether Type B progresses to Type C LAA. Patients with Type B or Type C LAA may have airflow limitation and decrease of diffusion capacity in pulmonary function tests.

It is not clearly explained the pathogenesis how these differences of CLE lesions occurred. The pathogenesis of emphysema is related to chronic innate and adaptive inflammatory immune response to inhaled toxic particles and gases as a result of the smoking habit [14]. Several evidences suggested that inflammatory cells centered on these airways and would destroy respiratory bronchioles and alveoli [15, 16]. Accumulations of pigmented macrophages in the alveolar spaces, thickening of the alveolar walls with inflammatory cells or organizing alveolitis in CLE lesions were also reported [14, 17]. In this study, we observed carbon deposits in bronchiolar and/or alveolar structures in cut surface of the inflated-fixed lungs, where pathologically accompanied with destruction of terminal and/or respiratory bronchioles and/or alveoli. These findings suggest that deposited particles or gases cause inflammation in bronchiolar wall and their marginal alveoli, resulting destruction of them in a secondary lobule.

We think that the differences of LAA type depend on the deposit sites of smoking particles or gases in a secondary lobule. The particles deposited around bronchioles cause airway inflammation, resulting in dilatation and destruction 


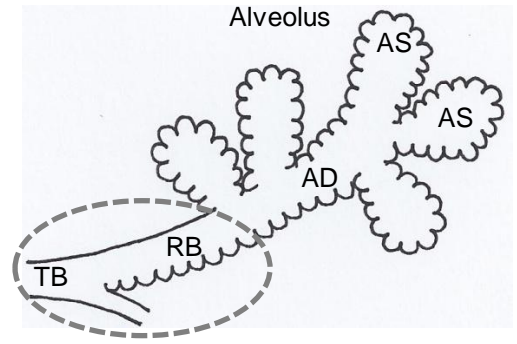

Type A

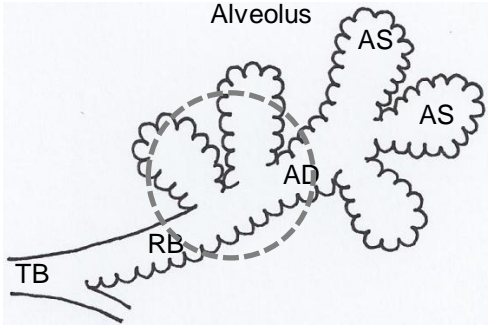

Type B

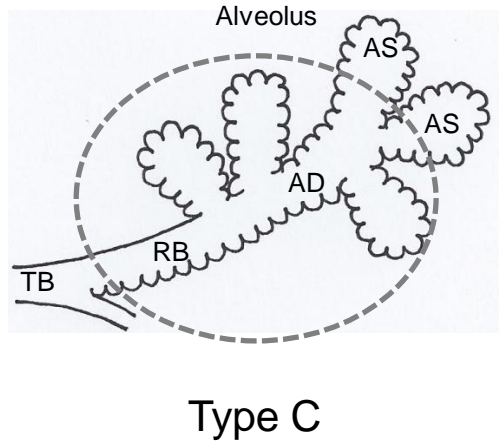

Fig. (3). Affected sites of Type A, Type B and Type C LAA. These diagrams show the parts of secondary lobule that are affected in different types of emphysema. The dotted circle demonstrates mainly dilated or destructed sites in bronchiolar and alveolar structures. TB: terminal bronchiole, RB: respiratory bronchiole. AD: alveolar duct, AS: alveolar sac.

of terminal and/or respiratory bronchiole. These morphological changes are recognized as Type A LAA in CT. Similarly, inhaled gases or very fine particles cause inflammation in alveolar duct or alveolar sac, resulting in destruction of proximal alveolar ducts in each secondary lobule. These changes lead to recognition of Type B LAA in CT. Moreover, when these destructions occur in distal alveolar ducts in each several secondary lobules and coalesce with each other, they may lead to recognition of Type $\mathrm{C}$ LAA. As shown in Fig. (3), the LAA types were thought to reflect affected sites in a secondary lobule in CLE.

Our study had limitations. First, the relationship between early and advanced lesions in CLE is not clear, because CLE lesions in inflated-fixed lungs demonstrate early stage. The relationship among Type A, Type B and Type C LAA were also unidentified. Second, the relationship between LAA types and respiratory function tests are not examined. Our study was performed in patients with undergoing operation or autopsy, which were not able to compare with pulmonary function tests. Application of our LAA classification to HRCT of patients with CLE might be a next step. Examination of relationship between LAA types and pulmonary function tests will be needed.

\section{CONFLICT OF INTEREST}

The authors confirm that this article content has no conflict of interest.

\section{ACKNOWLEDGEMENTS}

All authors thank Dr. Masahiko Yamagishi (Department of Internal Medicine, Sapporo Kojinkai Hospital) for his useful suggestions.

\section{REFERENCES}

[1] Rabe KF, Hurd S, Anzueto A, et al. Global strategy for the diagnosis, management and prevention of chronic obstructive lung disease. Am J Respir Crit Care Med 2007; 176: 532-55.

[2] Takahashi M, Fukuoka J, Nitta N, et al. Imaging of pulmonary emphysema: a pictorial review. Int J COPD 2008; 3: 193-204.
[3] Pipavath SN, Schmidt RA, Takasugi JE, et al. Chronic obstructive pulmonary disease: radiology-pathology correlation. J Thorac Imaging 2009; 24: 171-80.

[4] Copley SJ, Wells AU, Muller NL, et al. Thin-section CT in obstructive pulmonary disease: discriminatory value. Radiology 2002; 223: 812-9.

[5] Murata K, Itoh H, Todo G, et al. Centrilobular lesions of the lung: demonstration by high-resolution CT and pathologic correlation. Radiology 1986; 161: 641-5.

[6] Murata K, Khan A, Herman PG. Pulmonary parenchymal disease: evaluation with high-resolution CT. Radiology 1989; 170: 629-35.

[7] Foster WL, Pratt PC, Roggli VL, et al. Centrilobular emphysema: CT-pathologic correlation. Radiology 1986; 159: 27-32.

[8] Foster WL, Gimenez EI, Roubidoux MA, et al. The emphysemas: radiologic-pathologic correlations. Radiographics 1993; 13: 31128.

[9] Itoh $\mathrm{H}$, Tokunaga $\mathrm{S}$, Asamoto $\mathrm{H}$, et al. Radiologic-pathologic correlations of small nodules with special reference to peribronchiolar nodules. Am J Roentogenol 1978; 130: 223-31.

[10] Nishimura K, Kitaichi M, Izumi T, et al. Diffuse panbronchiolitis: correlation of high-resolution $\mathrm{CT}$ and pathologic findings. Radiology 1992; 184: 779-85.

[11] Markarian B, Dailey ET. Preparation of inflated lung specimens. In: Groskin SA, Ed. Heitzman's the lung, radiologic-pathologic correlations. $3^{\text {rd }}$ ed. St. Louis: Mosby 1993; pp. 4-12.

[12] Reid L. The secondary lobule in the adult human lung, with special reference to its appearance in bronchograms. Thorax 1958; 13 $110-5$.

[13] Katzenstein AA, Askin FB. Emphysema and small airway disease, Nonspecific inflammatory and destructive diseases of the lung. In Zorab R, Ed. Surgical pathology of non-neoplastic lung disease. $2^{\text {nd }}$ ed. Philadelphia: W.B. Saunders Co, 1990; pp. 547-50.

[14] Remy-Jardan M, Remy J, Gosselin B, et al. Lung parenchymal changes secondary to cigarette smoking: pathologic-CT correlations. Radiology 1993; 186: 643-51.

[15] Ratamales I, Elliott WM, Meshi B, et al. Amplification of inflammation in emphysema and its association with latent adenoviral infection. Am J Respir Crit Care Med 2001; 164: 46973.

[16] Turato G, Zuin R, Miniati M, et al. Airway inflammation in severe chronic obstructive pulmonary disease. Relationship with lung function and radiologic emphysema. Am J Respir Crit Care Med 2002; 166: 105-10.

[17] Remy-Jardan M, Remy J, Boulenguez C, et al. Morphologic effects of cigarette smoking on airways and pulmonary parenchyma in healthy adult volunteers: CT evaluation and correlation with pulmonary function tests. Radiology 1993; 186: 107-15. 